\title{
Research on intelligent prevention and control of COVID-19 in China's urban rail transit based on artificial intelligence and big data
}

\author{
Qi Liu ${ }^{\mathrm{a}, *}$ and Zhenzhen Huang ${ }^{\mathrm{b}, \mathrm{c}}$ \\ ${ }^{a}$ Urban Rail Transit Security Department, Railway Police College, Zhengzhou, Henan, China \\ ${ }^{\mathrm{b}}$ Railway Police Department, Railway Police College, Zhengzhou, Henan, China \\ ${ }^{\mathrm{c}}$ Police Sports Department, Railway Police College, Zhengzhou, Henan, China
}

\begin{abstract}
Since December 2019, the outbreak of novel coronavirus pneumonia has brought great challenges to global public health, which is the most serious epidemic over the past hundred years. The urban rail transit is an important part of public transport in large cities with characteristic of intensive passengers and confined space, which is easy to become viral infection intermediary. In order to prevent and control the situation of the epidemic, the police's public security department for urban rail transit and the urban rail transit operation company have established a three-layer filter network, which is composed of safety inspection, patrol and temporary interrogation, and intelligent police service, and this network implements the deep learning technology to identify key persons, prohibited luggage, and the body temperature of passengers. For the problem of uncertainty in total passenger flow and its density, this paper proposes a method for re-establishing the passenger flow model to focus on data monitoring, and resetting the threshold value of alarm to control the passenger density. In view of the difficulty of passenger identification caused by mask during the epidemic, this paper proposes a systematic schema of timely adjusting face recognition algorithm, modifying the alarm threshold, using iris recognition system, carrying out information collision comparison, deep mining and intelligent judging, which discover the high-risk groups of epidemic prevention and control in time. China's police's public security department for urban rail transit aims at prevention of virus input, infection, riot, fake new, scientific prevention and control, and has made precise policy implementation to hold urban rail transit's covid-19 intelligent prevention and control work, finally won the battle and effectively guaranteed the people's life safety and health.
\end{abstract}

Keywords: COVID-19, epidemic prevention and control, urban rail transit, intelligent police, big data, deep learning

\section{Introduction}

In December 2019, a number of cases of unknown pneumonia in Wuhan China were identified as acute respiratory infectious disease caused by a kind of

\footnotetext{
*Corresponding author. Qi Liu, Urban Rail Transit Security Department, Railway Police College, 450053 Zhengzhou, Henan, China. E-mail: liuqi@rpc.edu.cn.
}

novel coronavirus [1]. In February 11, 2020, this novel coronavirus pneumonia was named Corona Virus Disease 2019 (COVID-19) by World Health Organization (WHO) [2, 3]. In the press conference for COVID-19 held by WHO in June 8, 2020, the director general of the WHO Tedros Adhanom Ghebreyesus said that nearly 7 million cases report has been received, and the global epidemic has been deteriorating, with over 7.085 million cases of 


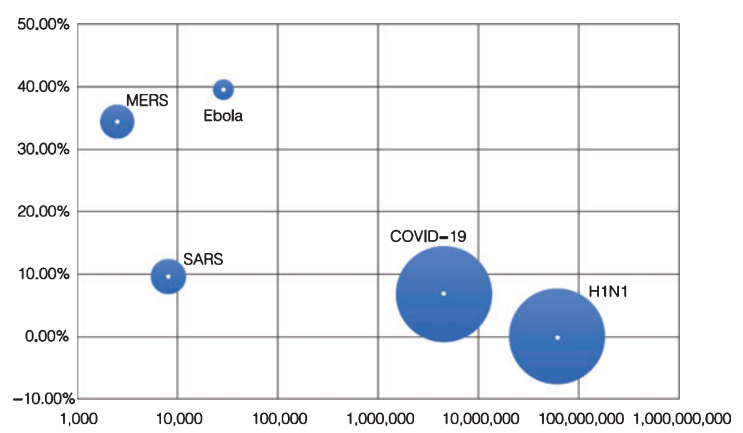

Fig. 1. Comparison of the number epidemicing countries, cumulative confirmed cases and fatality rate of major epidemic in the 21 st century.

newly diagnosed COVID-19 cases and 405 thousand cases died. Over 100 thousand cases have been reported during several days, and the day with the most cases is June 7, 2020, over 136 thousand cases added $[4,5]$.

The COVID-19 is one of the most serious infectious diseases in the past century, which has affected nearly 7 billion people and more than 210 countries in 3 months, and brought a big challenge to global public health. It has been the most serious epidemic already since the Spanish influenza in 1918. As shown in Fig. 1, the COVID-19 epidemic and the previous major epidemics in the 21 st century have similarities and differences in terms of lethality and scope of infection, but COVID-19 epidemic rate far exceeds that of other epidemics [6, 7].

The urban rail transit is an important part of public transport in large cities. A total of 41 cities in mainland China have put urban rail transit into operation, with a total mileage of $6730.27 \mathrm{~km}$, accounting for $23.92 \%$ mileage in the world. At present, China's urban rail transit operating line length, operating stations, annual passenger volume, operation scale and passenger intensity are still increasing. The urban rail transit is characteristic of intensive passengers and confined space, which is easy to become viral infection intermediary. In order to stop epidemic, from January 23, 2020, prevention and control measures, such as suspending all the lines of urban rail transit, pausing some lines, closing some stations and adjusting the operation time or running interval, have been used in China, consequently a significant drop in passenger flow appeared. From January 23, 2020 to February 10, 2020, the average daily passenger volume of urban rail transit in China has decreased by $70 \% 90 \%$ compared with the same period last year.

Since the resumption of work and production on February 10, 2020, the average daily passenger vol- ume of urban rail transit has rebounded. Specifically, the passenger traffic volume of Beijing, Shanghai and Guangzhou has increased by $72 \%, 99 \%$ and $48 \%$ respectively compared to that before the resumption, but it is still in a low level. On April 4, 2020, all 41 cities in China that have opened urban rail transit have resumed operation. The whole country has entered the critical period of epidemic prevention and control. The contradiction between the large passenger volume of urban rail transit and the strict prevention of passengers gathering during the epidemic prevention period is constantly highlighted. The detecting the temperature of passengers entering the station, and timely and reasonable transfer of suspected cases is required both. In view of these problems in COVID19 prevention and control, the police's public security department for urban rail transit comprehensively uses artificial intelligence and big data to prevent infection input, viral proliferation, riot and fake news, so as to improve the level of prevention and control and implement precise measures, put people's life safety and health first, carried out in-depth application of various intelligent public security prevention and control technologies under the epidemic situation, and achieved important results.

\section{Intelligent safety inspection and quarantine under high environmental risk condition}

The subway is the main form of urban rail transit, which has the characteristics of numerous stations, long lines, large prevention and control scale, dense passenger flow, closed space and poor air mobility [8]. Thus, it is very difficult to curb the epidemic and implement effective intervention measures for subway passengers. However, the COVID-19 aerosols can travel at a distance of up to 4.5 meters in a closed air-conditioned carriage, and it could float in the air in at least 30 minutes and causes infection $[9,10]$. The police public security department and the urban rail transit operation companies have established a three-layer public security prevention and control filter network to strengthen the security defense line of epidemic prevention and control.

\subsection{Smart security check}

Aiming at the problem of key passengers and prohibited luggage identification under epidemic situation, the police public security department use the 


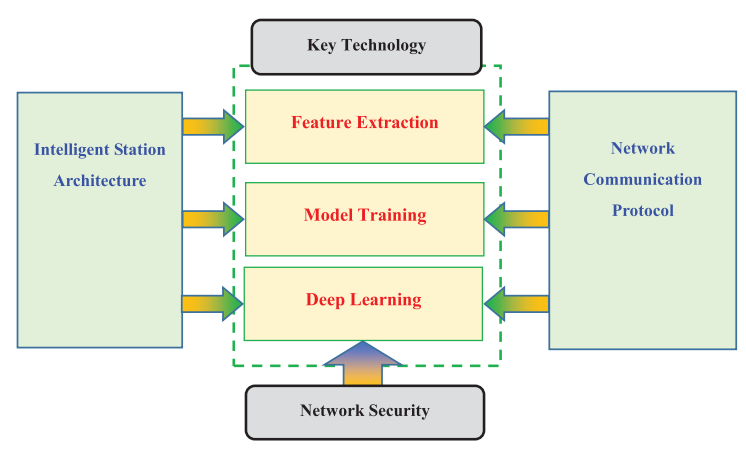

Fig. 2. Security inspection platform based on multidimensional information intelligence perception.

intelligent recognition technology of deep learning to aggregate the features of key persons in the database to mining data features of prohibited and restricted luggage, and continuously improve the accuracy and reliability of model detection with early warning capability through modeling training, test identification, strengthening and iterative updating Detection scope.

The public security department [11] and the urban rail transit operation company [12] will connect the intelligent station architecture, network communication protocol and network security into the security inspection management platform, and transfer the security inspection equipment, key public security personnel database, ticketing information system and credit data to establish a multi-dimensional information perception network system for accurate security inspection, as shown in Fig. 2.

\subsection{Quarantine and automatic monitoring}

Public security department and urban rail transit enterprise require passengers entering the station with masks and conduct mandatory body temperature detection at the security inspection office. Fixed thermal imagers are used in key stations with large passenger flow, while staff in stations with small passenger flow are required to wear protective clothing and use hand-held thermal imager or electronic thermometer to detect the temperature of passengers. The thermal imaging is shown in Fig. 3. At the same time, disinfection, ventilation and cleaning should be strengthened. In addition to mention routine methods, comprehensive and frequent disinfection should be carried out in public toilets, stair handrails, escalators, ticket machines, gate machines, security inspection equipment and other parts of the station, such as the

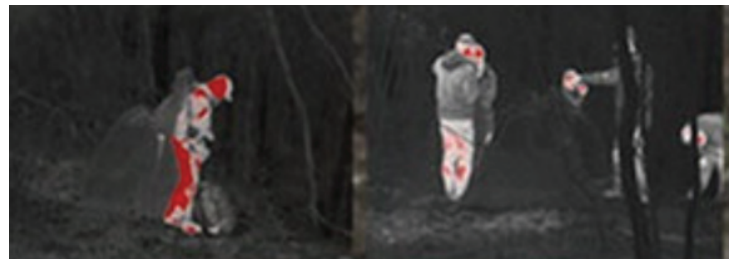

Fig. 3. Thermography.

handle inside the carriage, window glass, seat, door, air conditioner outlet, etc. The police public security department patrol and check the key hub stations, and check the track information of the household registration personnel in the key epidemic areas, and transfer the risk personnel to the local health department for disposal.

\section{Forecast and control of uncertain passenger flow}

COVID-19 is a once-in-a-century global epidemic disease. This major health event disrupts the passenger flow pattern of Spring Festival transportation in China, stops production and delays resumption, which brings people's mental stress, as well as the civil aviation, railway and long-distance coach. Consequently, the uncertainty of total passenger flow and passenger flow density increases.

\subsection{Representation model of uncertainty}

The normal cloud model is an uncertain transformation model between qualitative concept and quantitative expression. The membership function of normal cloud comprehensively represents fuzziness (this and that property of boundary) and randomness (probability of occurrence), and combines them completely to form the mapping between qualitative and quantitative, as shown in Fig. 4. The membership degree $u_{i k}$ can be obtained by Equation (1).

$$
u_{i k}(l)=\frac{\left(1 /\left\|x_{i}-w_{k}(l)\right\|^{2}\right)^{1 / m-1}}{\sum_{i=1}^{q}\left(1 /\left\|x_{i}-w_{k}(l)\right\|^{2}\right)^{1 / m-1}}
$$

The probability density distribution function is shown in Equation (2). 


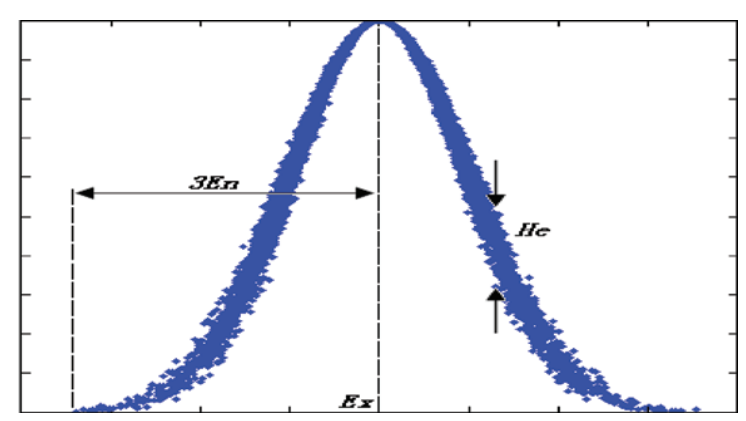

Fig. 4. Mathematical characteristics of normal cloud model.

$$
f_{\mathrm{x}}(x)=\int_{-\infty}^{\infty} \frac{1}{2 \pi H e|y|} \exp \left[-\frac{(x-E x)^{2}}{2 y^{2}}-\frac{(y-E n)^{2}}{2 H e^{2}}\right] d y
$$

\subsection{Calculation of passenger flow model}

The historical data before the epidemic is meaningless for the passenger flow prediction during the epidemic period. Since the resumption of production and work on February 10, 2020, it is difficult to predict and judge the peak passenger flow on the return journey of the Spring Festival. The practice of cancelling the traffic restriction and encouraging individual travel makes it more difficult to predict the passenger flow. The existing data are not enough to establish a scientific passenger flow prediction model, which makes it difficult to accurately predict the passenger flow, which make it impossible to do scientific prevention and control schema. Thus it is necessary to re-establish the passenger flow model for the epidemic period and recalculate the crosssection passenger flow and the number of people gathered in the station. Section passenger flow calculation method is shown in Equation (3).

$$
x_{i j}=\sum_{(r, s) \in W} \sum_{k \in R_{w}} \sum_{t \in\left(\left(T_{i}-t_{r-i}\right)-\left(T_{2}-t_{r-i}\right)\right)} f_{k}^{r s} \bullet \delta_{i j, k}^{r s}, \forall i, j
$$

The number of passengers gathered in the station can be obtained by Equation (4).

$$
\begin{gathered}
\operatorname{mum}_{i}(t)=\operatorname{mum}_{i}(t-1)+O_{i}(t)-D_{i}(t) \\
-f \bullet c+x_{j i}(t)+\left(\sum_{k-1}\left(x_{i}^{m, l}-x_{i}^{l, m}\right)\right)
\end{gathered}
$$

\subsection{Passenger flow prediction and control}

Passenger flow control is an organization measure to reduce the density of passenger in order to ensure operation safety or reduce the risk of cross infection.

During the epidemic period, urban rail transit operation companies increased big data monitoring, and paid close attention to passenger flow changes, dynamically adjusted the shift density of urban rail transit lines, strictly controlled the full load rate of cars, and reduced the risk of virus cross infection caused by dense passengers carriages [13]. Passenger flow control checkpoints are set up outside the station, station hall and carriage. According to the real-time passenger flow density, the speed of passengers entering the station and taking the bus is controlled. The passenger flow density of all parts is strictly controlled to reduce the risk of epidemic infection. By using the intelligent command platform, the passenger flow data model is rebuilt, the passenger flow warning threshold is reset, the passenger flow dynamic is paid attention to in real time, the peak value of return passenger flow is grasped, the police force scale are configurated according to passenger flow echelon, in order to strengthen the patrol, the control of passenger flow density of subway carriage, and adjust the departure frequency.

\section{Identification and traceability of key passengers}

Affected by the epidemic situation, passengers wear protective equipment such as masks, hats, goggles, etc., which makes it more difficult to collect face information and identify personnel. As a result, onsite police can not timely detect and deal with them according to face early warning, which brings great challenges to the public security prevention and control and anti-terrorism work of rail transit [14]. It is also difficult to trace the source of imported cases in urban rail transit without real name system, and it is extremely difficult to find close contacts.

\subsection{Adjust the face recognition algorithm}

Face recognition is a biometric recognition technology based on human facial feature recognition. At present, the application of face recognition technology in public security field mainly uses principal component analysis method, combing projection map, feature description matching method, 


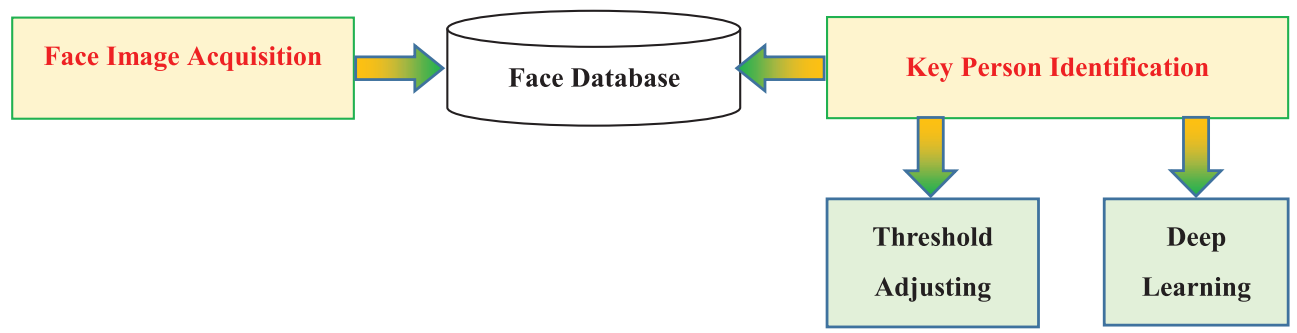

Fig. 5. Face recognition application system.

facial symmetry processing reduction method, threedimensional face similarity evaluation method of geodesic region, and construction of hidden Markov model to realize identity verification [15]. On situation that passengers wear mask causing face occlusion, it is necessary to adjust the face recognition algorithm in time and modify the alarm threshold. The threshold adjustment can be adjusted according to the situation of neighborhood points of pixels. The specific method is as follows Equation (5).

$$
T=\frac{1}{P} \sum_{i=0}^{P-1} g_{i} \times n \%
$$

As for the actual situation of epidemic prevention and control, information systems such as intelligent portrait profiling, passenger flow big data analysis, intelligent security inspection are necessary to be constructed, which integrate video face information, passenger information, security inspection information and implement information collision with comparison, deep mining and intelligent search and judgment, in order to identify high-epidemic groups in time. The video tracking and two-dimensional code of health registration is used to accurately track and trace the passengers, improving the accuracy of epidemic prevention and control, realizing the locking of suspicious people in massive information, as shown in Fig. 5.

\subsection{Iris recognition system}

During the epidemic period, the face was covered by masks, which made it difficult to identify the identity of passengers. Iris is the third visible biological feature after fingerprint and face, which can be quickly and accurately identified. The established iris recognition system can meet the requirements of urban rail transit passenger flow on epidemic prevention and control. The appearance of human eyes

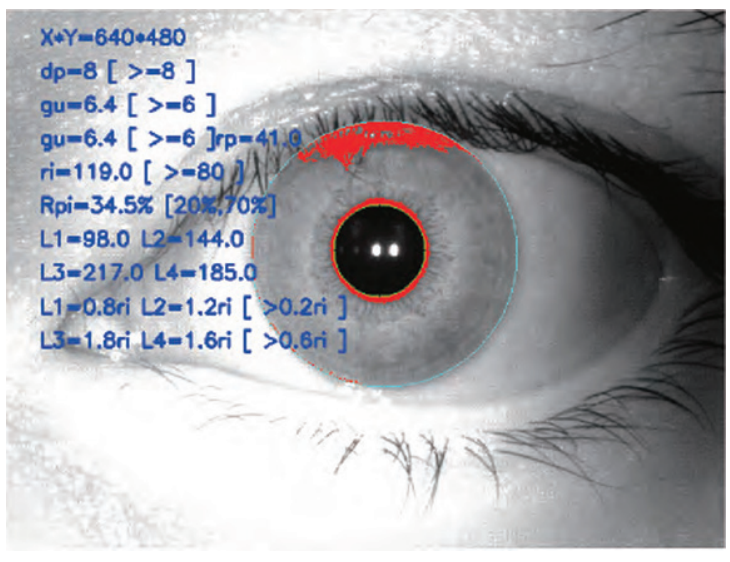

Fig. 6. Iris image quantization index.

is composed of sclera, iris and pupil, as shown in Fig. 6. Iris is a circular area between sclera and pupil, accounting for $65 \%$ of the total eye area, including abundant texture information such as concave spots, radial lines, pigment spots and other spots. The iris information of adults is permanent in human life.

The contrast between iris and sclera can be expressed by Equation (6).

$$
c t_{i s}=\left\{\begin{array}{cc}
\frac{\left|v_{s}-v_{i a}\right|}{v_{s}+v_{i a}-2 v_{p}} & \begin{array}{c}
v_{p} \geq v_{i a} \text { or } v_{p} \geq v_{s} \\
\text { Others }
\end{array}
\end{array}\right.
$$

The contrast of iris and pupil can be expressed by Equation (7).

$$
c t_{i p}=\frac{\left|v_{i b}-v_{p}\right|}{0.75 \times\left(20+v_{p}\right) v_{s}+\left|v_{i b}-v_{p}\right|} \times 100
$$

The contrast between iris and pupil should not be less than 30 .

Similar to fingerprint and face recognition, iris recognition system mainly includes three subsystems: iris image acquisition, iris image preprocessing, feature extraction and matching, as shown in Fig. 7. 


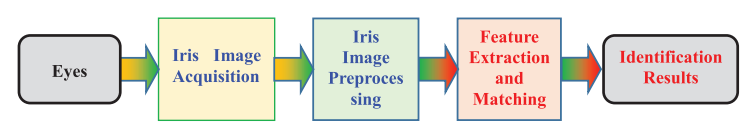

Fig. 7. Basic process of iris recognition.

\section{Conclusion}

The prevention and control of the most serious epidemic situation in the past 100 years should focus on the prevention of viral input and proliferation. The urban rail transit stations and vehicles are densely populated and the space is closed. It is a high-risk environment for epidemic infectious diseases, where is the difficulty of epidemic prevention and control. The police public security department for urban rail transit in China and the urban rail transit operation company have established a three-layer public security prevention and control filter network, composed of safety inspection, patrol and interrogation, and intelligent policing. The deep learning technology is used to strengthen the intelligent identification of key passengers and prohibited luggage. Both of them will carry out mandatory temperature test for passengers entering the station, and carry out comprehensive and frequent disinfection. In view of the uncertainty of the total passenger flow and the passenger flow density, the new passenger flow model during the epidemic period was established, the cross-section passenger flow and the number of people gathered in the station were recalculated, the big data monitoring was increased, and the passenger flow changes were closely monitored. The passenger flow control checkpoints were set up outside the station, the station hall and the carriages to control the speed of passengers entering the station and taking the bus and reset the passenger flow warning threshold. In view of the difficulty of face information collection and personnel identification caused by passengers with masks during the epidemic, timely adjust the face recognition algorithm, the alarm threshold modification, video face information collision, iris recognition system, collect iris images are used to discover highrisk groups in time, and comprehensively analyze their identity characteristics, activity trajectory and relationship network.

\section{Acknowledgments}

This paper is supported by Henan Provincial Research Foundation for Science and Technologi- cal Breakthroughs, China (Grant No. 182102210119) and Railway Police College Teaching Reform Research Projects (Grant No. JY2019004).

\section{References}

[1] A.E. Tarek Mohamed, S. James D. Recent progress and challenges in drug development against COVID-19 coronavirus (SARS-CoV-2)-an update on the status, Infection Genetics and Evolution 83(104327) (2020).

[2] E.J. Emanuel et al., Fair Allocation of Scarce Medical Resources in the Time of Covid-19, New England Journal of Medicine 382(21) (2020), 2049-2055.

[3] T. McEnery, C. Gough and R.W. Costello, COVID-19: Respiratory support outside the intensive care unit, The Lancet Respiratory Medicine 8(6) (2020), 538-539.

[4] C. Goldbaum, L. Rogers Cook, They Can't Afford to Quarantine. So They Brave the Subway, New York Times March $30,2020$.

[5] K.H. Hong, S.W. Lee, T.S. Kim, Guidelines for Laboratory Diagnosis of Coronavirus Disease 2019 (COVID-19) in Korea, Annals of Laboratory Medicine 40(5) (2020), 351-360.

[6] A.G. Cuevas, K. O'Brien and S. Saha, African American Experiences in Healthcare: 'I always feel like I'm getting skipped over,' Health Psychology 35(9) (2016): 987-995.

[7] W.K. Poornima and P. Ajnesh, The impossibility of social distancing among the urban poor: the case of an Indian slum in the times of COVID-19, Local Environment 25(5) (2020), 414-418.

[8] F. Yu, M. Thierry and S. Ted, Influence of wind and relative humidity on the social distancing effectiveness to prevent COVID-19 airborne transmission: A numerical study, Journal of Aerosol Science 147(105585) (2020), 1-19.

[9] S. Asadi, A. S. Wexler, C.D. Cappa, S. Barreda, N.M. Bouvier and W.D. Ristenpart, Aerosol emission and superemission during human speech increase with voice loudness, Scientific Reports 9(1) (2019), 1-10.

[10] N. van Doremalen, T. Bushmaker, D.H. Morris, M.G. Holbrook, A. Gamble, B.N. Williamson, et al., Aerosol and surface stability of SARS-CoV-2 as compared with SARSCoV-1, New England Journal of Medicine 382 (2020), 1564-1567.

[11] Q. Liu, Z.Z. Huang, H.Z. Xue, et al., Modelling Research on Tactical Coordination for the Minimum Combat Unit in Urban Rail Transit Environment, Journal of Environmental Protection and Ecology 19(03) (2018), 1065-1071.

[12] E. Knut, Emergency management training: Handling rich qualitative and quantitative data, Journal of Intelligent \& Fuzzy Systems 31(02) (2016), 939-948.

[13] X.B. Ding, Z.G. Liu, H. Hu, et al., Urban rail transit operation risks intelligent identification algorithm based on dispatching fault $\log$ data mining, Journal of Intelligent \& Fuzzy Systems 37(04) (2019), 4511-4522.

[14] K. Tony, UK senior police officer with COVID-19, Lancet Respiratory Medicine 8(5) (2020), 451-451.

[15] Q. Liu, S. Li and Z.Z. Huang, Research on the Fusion Method of Target Tracking in Minimum Combat Unit of Urban Rail Transit, Journal of Environmental Protection and Ecology 19(04) (2018), 1704-1710. 